\title{
Causes, assessment and management of cesarean scar pregnancy
}

\begin{abstract}
Cesarean scar pregnancy (CSP) is on the rise worldwide due to the increasing rate of cesarean sections. It has been attributed to life threatening complications if not diagnosed early and managed aggressively. CSP is still a rare diagnosis, as available literature is mostly observational case series and few random controlled trials (RCT) to date. Treatment of CSP should be evidence based and focus on prevention of severe complications, conservation of fertility and maintaining women's health and quality life. Despite this prominent increase, no universal treatment guidelines have been established till now, and the management of CSP in clinical practice is based on few available published literatures. Treatment should be individualized by using parameters such as hemodynamic status of the patient, beta-hCG levels, imaging features, the desire for future pregnancy and the surgeon's skill. Current data does not support expectant management. In this article, we aim to find the pathophysiology, clinical presentation, most appropriate methods of diagnosis and treatment of CSP.
\end{abstract}

Volume 2 Issue 5 - 2017

\author{
Lilian Onwonga, Sunita Thapa, Humphrey \\ Ochin, Wenjun Cheng \\ Department of Obstetrics and Gynecology, Nanjing Medical \\ University, China
} Correspondence: Department of Gynecology, First Affiliated
Hospital with Nanjing Medical University, 300 Guangzhou Road,
Jiangsu Province People's Hospital Nanjing 210029 China,Tel 8618724018533,Email wenjunchengdoc@163.com

Received: June 18,2017| Published: June 21, 2017

Keywords: ectopic pregnancy, previous cesarean scar, cesarean scar pregnancy

\section{Introduction}

Cesarean scar pregnancy is rare but potentially life threatening type of ectopic pregnancy. It is a condition in which the concepts implants in an iatrogenic cesarean scar in the uterus and is surrounded by uterine muscular fiber, scar tissue, and thin myometrium adjacent to the bladder. It can occur in women with a single previous cesarean delivery. CSP was first described by. ${ }^{1}$ The incidence of CSP has been reported to be 1:1800-1:2216 of normal pregnancies and accounts for $6.1 \%$ of all ectopic pregnancies. ${ }^{2-5}$ This rate is expected to rise in the future worldwide owing to increasing rates of cesarean deliveries and awareness of its pathology. During the last two decades ultrasonography diagnosis have improved and the techniques for uterine surgery has changed .In recent time the uterus is often closed in one layer, compared with the previous two-layer technique. These factors may play a role in the increasing prevalence of CSP. Vial et al. ${ }^{6}$ proposed two different types of CSPs; Endogenous CSP (CSP type I) and Exogenous CSP (CSP type II). CSP type I is a superficial implantation of the embryo on the cesarean scar (CS) which develop towards the cervicoisthmic space or the uterine cavity. CSP type II is a deep implantation of the embryo into a CS defect growing towards the bladder, infiltrating the uterine myometrium, and bulging from the uterine serosa. CSP type II may result in uterine rupture and hemorrhage during the first trimester of pregnancy. The above two types of CSP are easily differentiated by ultrasound or MRI examination. Due to the high risk of life-threatening complications including massive hemorrhages/shock, uterine rupture with the potential necessity for hysterectomy in worst case scenario and maternal death, CSP needs a prompt accurate and proper diagnosis and management. However, termination of pregnancy is generally recommended. Routine transvaginal ultrasonography has been recommended in early pregnancy for patients who have previously undergone a cesarean delivery. ${ }^{7-9}$

\section{The pathogenesis and risk factors of CSP}

The causes and mechanism of CSP is unclear, Jurkovic et al. ${ }^{3}$ hypothesized that the pathogenesis of CSP can be explained by the presence of uterine scar dehiscence and small scar defects after cesarean deliveries. A compromise of the deciduas basal is and the nitabuch fibrinoid layer, which normally constitutes a barrier preventing trophoblast invasion into the scarred myometrium of the lower anterior uterine segment enables the concepts to invade the myometrium through this microscopic dehiscence or defect in the scar. ${ }^{3}$ Such defects can also develop from the trauma of other uterine surgery for example; curettage, myomectomy, metroplasty, hysteroscopy and even manual removal of placenta. Seow et al. ${ }^{10,11}$ showed a possible correlation between intrauterine device, pelvic inflammatory disease and CSP. ${ }^{2}$ Recently Zhi-Da et al demonstrated that the increased expression of decidual integrin $\beta 3$ and Leukaemiainhibitory factor (LIF) in the cesarean scar might be associated with embryo implantation in cesarean scar, which might lead to a CSP; and the occurrence of CSP might be related to the changes of endometrial receptivity in local cesarean scar. $\mathbf{6 2}$ Information on any correlation between the number of previous caesarean sections, indication of the caesarean delivery, surgical technique and subsequent CSP are inadequate. . $^{3,9,10-13}$

\section{Clinical presentation}

CSP may present as early as 5-6 weeks 6 to 16 weeks 5 of gestational age. The clinical manifestations of CSP range from no symptoms (which can be incidentally detected by routine ultrasonographic examination) to amenorrhea, vaginal bleeding, abdominal discomfort, acute-onset lower abdominal pain, and even hypovolemic shock. In a review of 57 patients with CSP, $36.8 \%$ of the women were asymptomatic, $38.6 \%$ presented with painless vaginal bleeding, and only $24.6 \%$ presented with abdominal pain..$^{14}$ Acute onset of severe abdominal pain and profuse bleeding per vaginal may be a sign of impending uterine rupture and the uterus may be very tender on examination, while hemodynamic instability and shock implicates ruptured CSP5

\section{Diagnosis}

The diagnosis of CSP is based on a thorough history, including obstetric and surgical history, physical examination, laboratory and 
radiographic findings. The diagnosis of CSP is often difficult, and a false-negative diagnosis may result into major complications. A literature search identified 751 cases of CSP, 13.6\% had been misdiagnosed as either spontaneous abortions in progress, cervical pregnancies or low intrauterine pregnancies. Transvaginal ultrasound (TVUS) is the first-line diagnostic tool for CSP, following a positive chemical pregnancy test with serum human chorionic gonadotropin (beta-hCG). A report has shown a sensitivity of $86.4 \%{ }^{14}$ of TVUS combined with color, spectral, and power Doppler imaging. Threedimensional (3D) ultrasonography is also being increasingly used as it allows studying of a confined area in detail. ${ }^{15,16}$ The following diagnostic criteria have been proposed: empty uterus and cervical canal; development of the gestational sac or fetal pole with or without cardiac activity or identification of a mixed-echo mass in the anterior part of the cesarean scar

a. Very thin myometrium $(1-3 \mathrm{~mm})$ or an absence of healthy myometrium between the bladder wall and the sac/mass.

b. The gestational sac or mixed-echo mass being located toward either the cervicoisthmic space or the uterine cavity in CSP-I, or the infiltration of the gestational sac or mixed-echo mass into the myometrium and/or forming a bulge from the uterine serosal layer in CSP-II.

c. High velocity with low impedance peritrophoblastic vascular flow clearly surrounding the sac in Doppler examination. . $7,7,17,18$

Transabdominal ultrasound can also be used. However, transvaginal ultrasound is better due to its lower spatial resolution, fine details of placental implantation site, definition of embryonic/fetal as well as extraembryonic structures. The use of ultrasonography in diagnosing CSP has the advantages of being non-invasive, simple, and cheap. Magnetic resonance imaging (MRI) has been used as an adjunct to ultrasound scan, although not compulsory for the diagnosis. ${ }^{9,19,20}$ It is notable that MRI is a costly diagnostic technique which has a long acquisition time, and mostly used for cases where TVUS and color flow Doppler are inconclusive. ${ }^{22}$ Diagnostic hysteroscopy and diagnostic laparoscopy allows direct visualization and can be also therapeutic. ${ }^{20-22}$

\section{Differential diagnosis}

The differential diagnosis includes a cervicoisthmic pregnancy, or a spontaneous miscarriage in progress. A cervical pregnancy is extremely rare in patients with previous cesarean delivery. Therefore, the differential diagnosis of a cervical pregnancy is mainly in patients with no history of cesarean delivery, heavier bleeding, presence of a layer of healthy myometrium visible between the bladder and the gestational $\mathrm{sac}^{19}$ and gestational sac present within the cervix, giving an hour-glass shape to the uterus with a ballooned cervical canal on ultrasound..$^{2,17,21}$ In cases of a spontaneous miscarriage in progress, volume of bleeding is often greater due to detachment of a failed chorionic sac that is visibly seen in the cervical canal on TVUS, and on color flow Doppler, with the sac appearing avascular. ${ }^{23} \mathrm{~A}$ drastic fall in the serum $\beta$-hCG level is noticed in a miscarriage. Occasionally, CSP can be misdiagnosed as a trophoblastic tumour if ultrasound scan indicates no evidence of pregnancy within the uterus but detects high vascularised mixed echo mass infiltrating the myometrium in presence of high serum $\beta$-hCG level. Diagnosis of CSP is relatively easy and early in pregnancy, but as it advances, the disparity between CSP, cervical pregnancy and low implanted intrauterine pregnancy becomes more difficult. ${ }^{4,21}$

\section{Management}

Management of CSP is controversial, no universal treatment guidelines have been established, and its management in daily clinical practice is still based on the available data obtained from a limited number of patients. Not less than 30 primary therapeutic approaches towards the management of CSP have been published in literature. The treatment options such as expectant, medical, and surgical management or a combination of both have been proposed. ${ }^{12}$ There is no consensus on treatment strategy in relation to CSP type or thickness of the myometrium between the bladder and gestational sac. Systemic methotrexate, uterine artery embolization, dilatation and curettage $(\mathrm{D} \& \mathrm{C})$, and hysteroscopy are the most frequently adopted first-line approaches for CSP.

The aim of classifying CSP prior to treatment is to decrease the risk of adverse complications based on the characteristics of CSP types. In patients with CSP type I, the goal behind the choice of treatment is to evade prolonged procedures, with the aim of reducing complications and treatment failures. For patients with CSP type II, the preservation of patient fertility and a decreasing the risk of massive blood loss should be the priorities. ${ }^{7}$ Individualizing treatment according to the CSP subtype, gestational age, fetal viability, severity of symptoms, serum hCG levels and ultrasonography findings, yields substantial improvement in clinical outcome.

The prospect of expectant management is that the concepts will either be reabsorbed or expelled by itself against the risk of uterine rupture and hemorrhage or proceed to term. Available data on expectant management show that it is hardly ever successful, and when it does, the prognosis for an uneventful term pregnancy is poor with high cases of hysterectomies secondary to placenta previa/ accreta, uterine rupture or life-threatening massive hemorrhage. ${ }^{2,9,14,24}$ Therefore, termination of pregnancy in the first trimester is usually recommended. At this gestational age, the embryo is soft and fragile, the depth of placental implantation and the risk of invasion of the bladder are also considerably less than in late pregnancy. ${ }^{16}$

Systemic Methotrexate (MTX) administration is the least invasive treatment and has been widely used for stable patients but not effective for CSP compared with when used for tubal ectopic pregnancies. CSP has been shown to respond well in a dose of $50 \mathrm{mg} / \mathrm{m}^{2}$ and beta-HCG level of less than $5000 \mathrm{mIU} / \mathrm{ml} .{ }^{15-22}$ This is appropriate for a woman who is not in pain, haemodynamically stable with an unruptured CSP of $<8$ weeks of gestation and a myometrial thickness $<2 \mathrm{~mm}$ between the CSP and the bladder. ${ }^{9,25}$ Nevertheless, alternative treatment interventions are necessary when the $\beta-\mathrm{hCG}$ level is $>6000 \mathrm{mIU} / \mathrm{mL}$. MTX has a short half-life of 10 hours. Multiple doses of MTX have a higher success rate of about $75 \%$ than single dose of MTX. The advantages of this treatment include preservation of fertility and evading the need for surgery with its associated risks and complications.

However, its effect to stop the heart beats requires many days, which may not happen. Therefore, a long period of follow-up for beta-hCG to return to normal levels and for the conceptus to resolve completely is needed. It may also lead to further growth of the fetus as well as vascularization of the sac, thereby prolonging the of time treatment. A subsequent secondary treatment with a possibly high rate of complication may jeopardize the patient.

It can be debated whether systemic MTX is the treatment of choice as some investigators advocate for local injection of MTX 
into the gestational sac because, the impaired vascularization of the fibrous tissue surrounding the sac may impair the penetration of sufficient MTX into the fetal sac. In systematic review of 1647 patients, 559 patients treated with Systemic MTX only $8.7 \%$ of cases were successful. ${ }^{26}$ Another review of 751 showed a $62.1 \%$ rate of complication when MTX was used alone. ${ }^{12}$ Secondary treatment was dilatation and curettage, uterine artery embolization, hysteroscopy, and transvaginal sonography-guided intragestational MTX .Systemic MTX as a single treatment of choice should generally be avoided. Multiple doses of systemic MTX may cause side effects such as nausea, vomiting, elevated hepatic enzymes, and bone marrow suppression.

Direct local injection of methotrexate into the amniotic cavity of a CSP under ultrasound guidance produce excellent outcomes as it achieves a high concentration locally and therefore interrupts the pregnancy more rapidly than systemic administration of the drug. But normalization of hCG levels and shrinkage of the sac still takes long with this treatment modality. Besides, this route has no added advantage over systemic administration with regard to serum drug level and systemic toxicity. Other feticide/embyrocides that have been used include potassium chloride, ${ }^{19}$ hyperosmolar glucose ${ }^{20}$ and crystalline trichosanthin .Combined regimens have been described by many authors including; injecting potassium chloride into the gestational sac followed by methotrexate, ${ }^{27}$ simultaneous intravenous and intra-amniotic injections of MTX. ${ }^{28}$ Complication rate is about $9.6 \%{ }^{12,18}$

Uterine artery embolization (UAE) is another nonsurgical treatment for CSP used as a primary treatment but should be used sparingly or not at all. A recently published expert review stated that UAE alone had a complication rate of $80 \%$, and thus, should be used as an auxiliary method for uncomplicated cases of CSP. ${ }^{12,26}$ Among CSP patients treated with UEA, $63-73 \%$ require curettage to stop vaginal bleeding or to remove the retained products of conception. ${ }^{8,29,30} \mathrm{UAE}$ has a number of advantages such as blockage of blood supply to the gestational sac, which causes embryo ischemia, hypoxia, atrophy and finally necrosis, and at the same time reduces the risk of bleeding during curettage. However, in the case of excessive bleeding, UAE can accurately detect and embolize the pelvic arteries to stop bleeding. As an embolic agent, gelatin sponge can embolize arteries effectively and at the same time be absorbed naturally 14-21 days later, so that the blood flow of the uterus can recover and uterine function is not affected. ${ }^{31-34}$ In view of these benefits of UAE, combined therapeutic approach should be considered in order to achieve optimal results.

Uterine curettage as a primary treatment for CSP can lead to severe hemorrhage that resulted from gestational sac rupture and myometrial disruption. The vessels exposed by curettage bleed because the thin scar tissue is unable to contract and contain the bleeding. Arslan et al. ${ }^{35}$ described the first case of CSP that was successfully treated with $\mathrm{D} \& \mathrm{C}$ alone, but failed with complications in some other cases. These patients were subsequently treated with different methods such as hysterectomy, systemic MTX or excision of the mass. Some authors argue for suction curettage under ultrasound guidance in selected cases of gestational age $\leq 7$ weeks and myometrial thickness $\geq 3.5$ $\mathrm{mm}$ anterior to the CSP. ${ }^{35,36}$ Prolonged beta-hCG follow up can be a major drawback.A recent review demonstrated D\&C alone treatment of CSP with complication rate of $63 \%,{ }^{12}$ Therefore, it would be lucid to avoid $\mathrm{D} \& \mathrm{C}$ as a first-line approach. Additionally, $\mathrm{D} \& \mathrm{C}$ as a firstline approach is associated with infertility and poor obstetric outcome irrespective of whether it is successful or not. ${ }^{12}$
Successful treatment of CSP by operative hysteroscopy and suction curettage has been described in various studies. ${ }^{39-43}$ A systematic review demonstrated hysteroscopy as an optimal first-line approach for CSP with a lower complication rate (18.2\%). It can be utilized for the CSP that grows inwards toward the uterine cavity. Hysteroscopy is a minimally invasive technique conducted under direct visualization or Transabdominal ultrasound. The gestational sac is dissected free of the uterine wall through a natural orifice, and hemostasis can be achieved with electro coagulation. A balloon catheter may be placed postoperatively for compression hemostasis and wound surface drainage. Other advantages of this technique over systemic MTX and UAE are the avoidance of toxicity, shorter duration of resolution, and rapid return to fertility. However, the procedure requires general anesthesia, well trained personnel, and operative equipment

Surgical intervention is another reliable treatment option for CSP. Hysterotomy with either laparotomy, laparoscopy and transvaginal route is quite successful with low failure rates. A systematic review ${ }^{44}$ demonstrated that hysterotomy successfully treated $92 \%$ of the reviewed CSP cases, and hysterectomy was required in only $2 \%$ of the cases. Moreover, the ability to achieve a subsequent term pregnancy was found to be related to successful hysterotomy. Surgical treatment has a number of advantages such as one-time lesion clearance and instantaneous repair of the scar, removes the microtubular tracts and thus reduces the risk of recurrence followed by a rapid return of the beta-hCG to normal level within 1-2 weeks, preservation of patient fertility, and no undesirable effect on menstruation. ${ }^{6,9,20}$ Laparotomy followed by wedge resection of the lesion should be considered in women who present too late or if facilities and expertise for operative endoscopy are not available. Laparotomy is necessary in cases where uterine rupture is established or strongly suspected. This approach, however, inflicts a larger surgical wound, longer hospital stay and recovery time; with an increased risk of a future placenta praevia/accrete. ${ }^{45}$ Laparoscopic wedge resection of CSP is justified in hemodynamically stable women who have deeply implanted gestational sacs growing toward the abdominal cavity and bladder. Reports suggest that laparoscopic evacuation of CSP is a safe and less time-consuming procedure but requires operative skills and equipment. ${ }^{13,31,45,46}$ Transvaginal surgery is a novel minimally invasive therapy best for CSP Type II. A retrospective analysis of 25 patients with CSP Type II conducted who received transvaginal surgical treatments had successful surgery, without intraoperative or postoperative complications. ${ }^{47}$ Robotic assisted laparoscopic removal of residual cesarean ectopic pregnancy was also reported by Schmitt et al. ${ }^{48}$

\section{High-intensity focused ultrasound ablation (HIFU)}

This procedure can be performed with ablation alone or in combination with hysteroscopic D\&C. ${ }^{44}$ The initial procedure is performed under conscious sedation. A transducer produces the therapeutic energy required. Real-time ultrasound is used to target the area of the gestational sac and monitor the response. Additional D\&C is performed under general anesthesia. This novel treatment modality described in two high-quality case series had a success rate of $100 \%$ with no complications and no additional treatment warranted. ${ }^{49-50}$ Since systemic MTX injections, dilatation and curettage, and UAE are treatments that have all demonstrated high complication rates previously, combining therapeutic approaches should be considered when selecting the treatment options. The following Combinations have been described in literature, with improved outcome and are more frequently used at present. 


\section{A. UAE+ D\&C, without MTX.}

Overall this treatment modality was efficient with additional treatment needed in only $6.4 \%$ and severe complications seen in $3.4 \% .{ }^{23}$ A systematic review by Timor-Tritsch et al demonstrated a complication rate of $29.5 \% .^{12}$

\section{B. $\mathrm{UAE}+\mathrm{D} \& \mathrm{C}+\mathrm{MTX}$}

Additional treatment was needed in $31.4 \%$ because of treatment failure. ${ }^{23}$

\section{C. $\mathrm{UAE}+\mathrm{D} \& \mathrm{C}+$ Hysteroscopy}

This modality has a high success rate of $95.4 \%$ and a very low complication rate of $1.2 \% .^{23}$

D. Systemic MTX + D\&C

Intramuscular methotrexate and $\mathrm{D} \& \mathrm{C}$ in combination had a complication rate of $86 \% .^{12}$

\section{E. UAE+ hysteroscopic surgery}

In a retrospective study of 67 patients with exogenous CSP by who underwent selective UAE combined with hysteroscopic surgery, treatment was successful without complications when the uterine scar tissue thickness was $>3 \mathrm{~mm}$. For uterine scar thickness $<3 \mathrm{~mm}, 3$ cases required secondary laparoscopic or open surgery, after surgery, 7 patients received a second hysteroscopic operation. ${ }^{49}$

\section{F. Intra-arterial MTX+UAE}

followed by combined laparoscopy and hysteroscopy. Intra-arterial MTX + UAE + combined laparoscopy and hysteroscopy 25 patients, $100 \%$ success rate. Blood loss less than $200 \mathrm{ml} .^{50}$

\section{G. UAE combined with local MTX or systemic MTX}

UAE combined with local MTX infusion has been used extensively in recent years, and it has been suggested that the effects of MTX are synergistic to UAE..$^{50,51}$

\section{H. Systemic MTX+hysteroscopy}

5 patients in the review by Timor-Tritsch et al, all had complications. ${ }^{12}$

\section{Hysterectomy}

Although hysterectomies have been reported in the literature as a primary procedure in a few cases, it is generally recommended as the last resort when other treatment modalities fail. This shows that CSP is a potentially serious condition despite advances in many of the diagnostic techniques and therapeutic measures. ${ }^{2,45}$ Risk of recurrence. The risk of reoccurrence is about $1 \% \cdot{ }^{53-59}$

\section{Conclusion}

CSP are rare form of ectopic pregnancies that can result into life threatening complications if not diagnosed early and managed aggressively. The most efficient and safe treatment modality for CSP is yet to be determined, but five approaches are recommended depending on availability, hemodynamic status of the patient, betahCG levels, imaging features, the desire for future pregnancy and the surgeon's skill . Systemic MTX, UAE and D\&C as a single treatment should be avoided due to the high complication rate. ${ }^{60-72}$

\section{Acknowledgements}

None.

\section{Conflict of interest}

Author declares that there is no conflict of interest.

\section{References}

1. Larsen JV, Solomon MH. Pregnancy in a uterine scar sacculus-an unusual cause of postabortal haemorrhage. A case report. S Afr Med J. 1978;53(4):142-143.

2. Seow KM. Ultrasound Diagnosis of a Pregnancy in a Cesarean Section Scar. Ultrasound Obstet Gynecol. 2001;18(5):547-549.

3. Jurkovic D, Hillaby K, Woelfer B, et al. First-trimester diagnosis and management of pregnancies implanted into the lower uterine segment Cesarean section scar. Ultrasound Obstet Gynecol. 2003;21(3):220-227.

4. Shen L. Bilateral uterine artery chemoembolization with methotrexate for cesarean scar pregnancy. Am J Obstet Gynecol. 2012;207(5):386. e1-386.e6.

5. Aniela Angerame Yela, Nathalia Marchiani. Conservative management of ectopic pregnancy in cesarean scar: case report. Rev Bras Ginecol Obstet. 2013;35(5):233-237.

6. Vial Y, Petignat P, Hohlfeld P. Pregnancy in a cesarean scar. Ultrasound Obstet Gynecol. 2000;16(6):592-593.

7. Li Y, Wang W, Yang $\mathrm{T}$, et al. Incorporating uterine artery embolization in the treatment of cesarean scar pregnancy following diagnostic ultrasonography. Int J Gynaecol Obstet. 2016;134(2):202-207.

8. Lian F, Wang Y, Chen W, et al. Uterine artery embolization with local methotrexate and systemic methotrexate for treatment of cesarean scar pregnancy with different ultrasonographic pattern. Cardiovasc Intervent Radiol. 2012;35(2):286-291.

9. Maymon R, Halperin R, Mendlovic S, et al. Ectopic pregnancies in Caesarean section scars: the 8 year experience of one medical centre. Hum Reprod. 2004;19(2):278-284.

10. Fait G, Goyert G, Sundareson A, et al. Intramural pregnancy with fetal survival: case history and discussion of etiologic factors. Obstet Gynecol. 1987;70(3 pt 2):472-474.

11. Chuang J, Seow KM, Cheng WC, et al. Conservative treatment of ectopic pregnancy in a caesarean section scar. BJOG. 2003;110:869-870.

12. Timor-Tritsch IE, Monteagudo A. Unforeseen consequences of the increasing rate of cesarean deliveries: early placenta accreta and cesarean section scar pregnancy; a review. Am J Obstet Gynecol. 2012;207(1):14-29.

13. Wang YL, Su TH, Chen HS. Laparoscopic management of an ectopic pregnancy in a lower segment of caesarean scar: a review and case report. J Minim Invasive Gynecol. 2005;12(1):73-79.

14. Rotas MA, Haberman S, Levgur M. Cesarean scar ectopic pregnancies: etiology, diagnosis, and management. Obstet Gynecol. 2006;107(6):1373-1381.

15. Wang G, Liu X, Bi F, et al. Evaluation of the efficacy of laparoscopic resection for the management of exogenous cesarean scar pregnancy. Fertil Steril. 2014;101(5):1501-1507.

16. Koplay M, Dogan NU, Sivri M, et al. Ectopic Pregnancy in a Cesarean Section Scar: Successful Management Using Vacuum Aspiration under Laparoscopic Supervision-Mini Review of Current Literature. Case Rep Sur. 2016;2016:7460687. 
17. Einenkel J, Stumpp P, Kosling S, et al. A misdiagnosed case of caesarean scar pregnancy. Arch Gynecol Obstet. 2005;271(2):178-181.

18. Timor Tritsch IE, Monteagudo A, Santos R, et al. The diagnosis, treatment, and follow-up of cesarean scar pregnancy. Am J Obstet Gynecol. 2012;207(1):44.e1-44.e13.

19. Godin PA, Bassil S, Donnez J. An ectopic pregnancy developing in a previous caesarean section scar. Fertil Steril. 1997;67(2):398-400.

20. Roberts H, Kohlenber C, Lanzarone V, et al. Ectopic pregnancy in lower segment uterine scar. Aust N Z J Obstet Gynaecol. 1998;38(1):114-116.

21. Smith A, Finnoff JT. Diagnostic and interventional musculoskeletal ultrasound: part 2. Clinical applications. PM R. 2009;1(2):162-177.

22. Wang YL, Su TH, Chen HS. Laparoscopic management of an ectopic pregnancy in a lower segment cesarean section scar: a review and case report. J Minim Inv Gynecol. 2005;12(1):73-79.

23. Hofmann HM, Urdl W, Hofler H, et al. Cervical pregnancy: case reports and current concepts in diagnosis and treatment. Arch Gynecol Obstet. 1987;241(1):63-69.

24. Boza A, Boza B, Api M. Cesarean Scar Pregnancy Managed with Conservative Treatment. Iran J Med Sci. 2016;41(5):450-455.

25. Ravhon A, Ben-Chetrit A, Rabinowitz R, et al. Successful methotrexate treatment of a viable pregnancy within a thin uterine scar. Br J Obstet Gynaecol. 2017;104:628-629.

26. Birch Petersen K, Hoffmann E, Rifbjerg Larsen C, et al. Cesarean scar pregnancy: a systematic review of treatment studies. Fertil Steril. 2016;105(4):958-967.

27. Richard J Persadie, Anne Fortier, Richard G Stopps. Ectopic pregnancy in a caesarean scar: a case report. J Obstet Gynaecol Can 2005;27(12):1102-1106.

28. Ayoubi JM, Fanchin R, Meddoun M, et al. Conservative treatment of complicated cesarean scar pregnancy. Acta Obstet Gynecol Scand. 2001;80(5):469-470.

29. Wu X, Zhang X, Zhu J, et al. Caesarean scar pregnancy: comparative efficacy and safety of treatment by uterine artery chemoembolization and systemic methotrexate injection. Eur J Obstet Gynecol Reprod Biol. 2012;161(1):75-79.

30. Yang XY, Yu H, Li KM, et al. Uterine artery embolisation combined with local methotrexate for treatment of caesarean scar pregnancy. BJOG. 2010;117(8):990-996.

31. Sarin SN, Baarson C, Hanif S, et al. Uterine artery embolization, in Women's health in interventional radiology. Springer; 2012:3-36.

32. Van der Kooij SM, Bipat S, Hehenkamp WJ, et al. Uterine artery embolization versus surgery in the treatment of symptomatic fibroids: a systematic review and metaanalysis. AmJ Obstet Gynecol. 2011;205(4):317e1-317e18

33. Zhang B, Jiang ZB, Huang MS, et al. Uterine artery embolization combined with methotrexate in the treatment of cesarean scar pregnancy: results of a case series and review of the literature. J Vasc Interv Radiol. 2012;23(12):1582-1588

34. Qi F, Zhou W, Wang MF, et al. Uterine artery embolization with and without local methotrexate infusion for the treatment of cesarean scar pregnancy. Taiwan J Obstet Gynecol. 2015;54(4):376-380

35. Arslan M, Pata O, Dilek TU, et al. Treatment of viable caesarean scar ectopic pregnancy with suction curettage. Int J Gynecol Obstet. 2005;89:163-166.

36. Yang XY, Yu H, Li KM, et al. Uterine artery embolization combined with local methotrexate for treatment of caesarean scar pregnancy. BJOG. 2010;117(8):990-996.
37. Wang CJ, Yuen LT, Chao AS, et al. Caesarean scar pregnancy successfully treated by operative hysteroscopy and suction curettage. BJOG. 2005;112(6):839-840.

38. Deans R, Abbot J. Hysteroscopic management of cesarean scar ectopic pregnancy. Fertil Steril. 2010;93(6):1735-1740.

39. Chao A, Wang TH, Wang CJ, et al. Hysteroscopic management of caesarean scar pregnancy after unsuccessful methotrexate treatment. $J \mathrm{Mi}$ nim Invasive Gynecol. 2005;12(4):374-376.

40. Robinson JK, Dayal MB, Gindoff P, et al. A novel surgical treatment for caesarean scar pregnancy: laparoscopically assisted operative hysteroscopy. Fertil Steril. 2009;92(6):1497.e13-1497.e16.

41. Li H, Guo HY, Han JS, et al. Endoscopic treatment of ectopic pregnancy in a cesarean scar. J Minim Invasive Gynecol. 2011;18(1):31-35.

42. Chang Y, Kay N, Chen YH, et al. Resectoscopic treatment of ectopic pregnancy in previous caesarean delivery scar defect with vasopressin injection. Fertil Steril. 2011;96(2):e80-e82.

43. Wang CJ, Chao AS, Yuen LT, et al. Endoscopic management of caesarean scar pregnancy. Fertil Steril. 2006;85(2):494-497.

44. Kanat-Pektas M, Bodur S, Dundar O, et al. Systematic review: what is the best first-line approach for cesarean section ectopic pregnancy? Taiwan J Obstet Gynecol. 2016;55(2):263-269.

45. Lee CL, Wang CJ, Chao A, et al. Laparoscopic management of an ectopic pregnancy in a previous caesarean section scar. Hum Reprod. 1999; 14(5):1234-1236.

46. Wang CJ, Yuen LT, Yen CF, et al. Three-dimensional power Doppler ultrasound diagnosis and laparoscopic management of a pregnancy in a previous caesarean scar. $J$ Laparoendosc Adv Surg Tech A 2004;14(6):399-402.

47. Zhang H, Shi J, Yang Y, et al. Transvaginal Surgical Management of Cesarean Scar Pregnancy II (CSP-II): An Analysis of 25 Cases. Med Sci Monit. 2015;21:3320-3326.

48. Schmitt A, Crochet P, Agostini A. Robotic-assisted laparoscopic treatment of residual ectopic pregnancy in a previous cesarean section scar: a case report. J Minim Invasive Gynecol. 2017;24(3):342-343.

49. Wang G, Liu X, Wang D, et al. Clinical analysis on selective uterine artery embolization combined with hysteroscopic surgery for exogenous cesarean scar pregnancy in 67 cases. Zhonghua Fu Chan Ke Za Zhi. 2015;50(8):576-581.

50. Zhu X, Deng X, Wan Y, et al. High-intensity focused ultrasound combined with suction curettage for the treatment of cesarean scar pregnancy. Medicine (Baltimore). 2015;94(18):e854

51. Xiao J, Zhang S, Wang F, et al. Cesarean scar pregnancy: noninvasive and effective treatment with high-intensity focused ultrasound. Am J Obstet Gynecol. 2014;211(4):356.e1-356.e7.

52. Lian F, Wang Y, Chen W, et al. Uterine artery embolization combined with local methotrexate and systemic methotrexate for treatment of cesarean scar pregnancy with different ultrasonographic pattern. Cardiovasc Intervent Radiol. 2012;35(2):286-291.

53. Yang XY, Yu H, Li KM, et al. Uterine artery embolisation combined with local methotrexate for treatment of caesarean scar pregnancy. BJOG. 2010;117(8):990-996.

54. McKenna DA, Poder L, Goldman M, et al. Role of sonography in the recognition, assessment, and treatment of cesarean scar ectopic pregnancies. J Ultrasound. 2008;27(5):779-783.

55. Peng KW, Lei Z, Xiao TH, et al. first trimester caesarean scar ectopic pregnancy evaluation using MRI. Clin Radiol. 2014;69(2):123-129. 
56. Zhang Y, Gu Y, Wang JM, et al. Analysis of cases with cesarean scar pregnancy. J Obstet Gynaecol Res. 2013;39(1):195-202.

57. Wang CJ, Chao AS, Yuen LT, et al. Endoscopic management of cesarean scar pregnancy. Fertil Steril. 2006;85(2):494-497.

58. Wang YL, Su TH, Chen HS. Operative laparoscopy for unruptured ectopic pregnancy in a caesarean scar. BJOG. 2006;113(9):1035-1038.

59. Lam PM, Lo KWK, Lau TK. Unsuccessful medical treatment of cesarean scar ectopic pregnancy with systemic methotrexate: a report of two cases. Acta Obstet Gynecol Scand. 2004;83(1):108-116.

60. Shufaro Y, Nadjari M. Implantation of a gestational sac in a cesarean section scar. Fertil Steril. 2001;75(6):1217.

61. Wang CJ, Chao AS, Yuen LT, et al. Endoscopic management of cesarean scar pregnancy. Fertil Steril. 2006;85(2):494-497.

62. Donnez O, Jadoul P, Squifflet J, et al. Laparoscopic repair of wide and deep uterine scar dehiscence after cesarean section. Fertil Steril. 2008;89(4):974-980.

63. Gubbini G, Centini G, Nascetti D, et al. Surgical hysteroscopic treatment of cesarean induced isthmocele in restoring fertility: prospective study. $J$ Minim Invasive Gynecol. 2011;18(2):234-237.

64. He M, Chen MH, Xie HZ, et al. Transvaginal removal of ectopic pregnancy tissue and repair of uterine defect for caesarean scar pregnancy. BJOG. 2011;118(9):1136-1139.
65. Klemm P, Koehler C, Mangler M, et al. Laparoscopic and vaginal repair of uterine scar dehiscence following cesarean section as detected by ultrasound. J Perinat Med. 2005;33(4):324-331.

66. Yalcinkaya TM, Akar ME, Kammire LD, et al. Robotic assisted laparoscopic repair of symptomatic cesarean scar defect: a report of two cases. $J$ Reprod Med. 2011;56(5-6):265-270.

67. Dodd JM, Anderson ER, Gates S. Surgical techniques for uterine incision and uterine closure at the time of caesarean section. Cochrane Database Syst Rev. 2008;16(3):CD004732.

68. Qian ZD, Weng Y, Wang CF, et al. Research on the expression of integrin $\beta 3$ and leukaemia inhibitory factor in the decidua of women with cesarean scar pregnancy. BMC pregnancy childbirth. 2017;17(1):84.

69. Gozdemir E, Simavli S. Cesarean Scar Pregnancy: Diagnosis and Treatment. J Nurs Care. 2014;3:182

70. He Y, Wu X, Zhu Q, et al. Combined laparoscopy and hysteroscopy vs. uterine curettage in the uterine artery embolization-based management of cesarean scar pregnancy: a retrospective cohort study. BMC Women's Health. 2014;14:116.

71. Qian ZD, Huang LL, Zhu XM. Curettage or operative hysteroscopy in the treatment of cesarean scar pregnancy. Arch Gynecol Obstet. 2015;292:1055-1061.

72. Yang XY, Yu H, Li KM, et al. Uterine artery embolisation combined with local methotrexate for treatment of caesarean scar pregnancy. BJOG. 2010;117(8):990-996. 